\title{
Sharp bounds of Fekete-Szegő functional for quasi-subordination class
}

\author{
Shashi Kant \\ Department of Mathematics, \\ Government Dungar College \\ Bikaner, India \\ email: drskant.2007@yahoo.com
}

\author{
Prem Pratap Vyas \\ Department of Mathematics, \\ Government Dungar College \\ Bikaner, India \\ email: prempratapvyas@gmail.com
}

\begin{abstract}
In the present paper, we introduce a certain subclass $\mathcal{K}_{\mathbf{q}}(\lambda, \gamma, \mathrm{h})$ of analytic functions by means of a quasi-subordination. Sharp bounds of the Fekete-Szegö functional for functions belonging to the class $\mathcal{K}_{q}(\lambda, \gamma, h)$ are obtained. The results presented in the paper give improved versions for the certain subclasses involving the quasi-subordination and majorization.
\end{abstract}

\section{Introduction and definitions}

Let $\mathcal{A}$ denote the family of normalized functions of the form

$$
f(z)=z+\sum_{n=2}^{\infty} a_{n} z^{n},
$$

which are analytic in the open unit disk $\mathbb{U}=\{z:|z|<1\}$. If $\mathrm{f} \in \mathcal{A}$ satisfies $\mathrm{f}\left(z_{1}\right) \neq \mathrm{f}\left(z_{2}\right)$ for any $z_{1} \in \mathbb{U}$ and $z_{2} \in \mathbb{U}$ with $z_{1} \neq z_{2}$, then $\mathrm{f}$ is said to be univalent in $\mathbb{U}$ and denoted by $f \in \mathcal{S}$.

2010 Mathematics Subject Classification: 30C45

Key words and phrases: univalent functions, subordination, quasi-subordination, FeketeSzegö coefficients 
Let $g$ and $f$ be two analytic functions in $\mathbb{U}$ then function $g$ is said to be subordinate to $f$ if there exists an analytic function $w$ in the unit disk $\mathbb{U}$ with $w(0)=0$ and $|w(z)|<1$ such that

$$
g(z)=f(w(z)) \quad(z \in \mathbb{U})
$$

We denote this subordination by $g \prec f$. In particular, if the $f$ is univalent in $\mathbb{U}$, the above subordination is equivalent to $g(0)=f(0)$ and $f(\mathbb{U}) \subset g(\mathbb{U})$. Further, function $g$ is said to be quasi-subordinate [18] to $f$ in the unit disk $\mathbb{U}$ if there exist the functions $w$ (with constant coefficient zero) and $\phi$ which are analytic and bounded by one in the unit disk $\mathbb{U}$ such that

$$
g(z)=\phi(z) f(w(z))
$$

and this is equivalent to

$$
\frac{g(z)}{\phi(z)} \prec f(z) \quad(z \in \mathbb{U}) .
$$

We denote this quasi-subordination by $\mathrm{g} \prec_{\mathrm{q}}$ f. It is observed that if $\phi(z)=1$ $(z \in \mathbb{U})$, then the quasi-subordination $\prec_{\mathrm{q}}$ become the usual subordination $\prec$, and for the function $w(z)=z \quad(z \in \mathbb{U})$, the quasi-subordination $\prec_{\mathrm{q}}$ become the majorization ' $\ll$ '. In this case

$$
g(z)=\phi(z) f(w(z)) \Rightarrow g(z) \ll f(z), \quad(z \in \mathbb{U}) .
$$

Some typical problems in geometric function theory are to study functionals made up of combinations of the coefficients of $f$. In 1933, Fekete and Szegö [5] obtained a sharp bound of the functional $\lambda a_{2}^{2}-a_{3}$, with real $\lambda(0 \leq \lambda \leq 1)$ for a univalent function $f$. Since then, the problem of finding the sharp bounds for this functional of any compact family of functions $f \in \mathcal{A}$ with any complex $\lambda$ is known as the classical Fekete-Szegö problem or inequality. Lawrence Zalcman posed a conjecture in 1960 that the coefficients of $\mathcal{S}$ satisfy the sharp inequality

$$
\left|a_{n}^{2}-a_{2 n-1}\right| \leq(n-1)^{2}, \quad n \geq 2 .
$$

More general versions of Zalcman conjecture have also been considered ([4, 12, 13, 14]) for the functional such as

$$
\lambda a_{n}^{2}-a_{2 n-1} \text { and } \lambda a_{m} a_{n}-a_{m+n-1}
$$


for certain positive value of $\lambda$. These functionals can be seen as generalizations of the Fekete-Szegő functional $\lambda a_{2}^{2}-a_{3}$. Several authors including [1]-[4], [9]$[15],[17,20]$ have investigated the Fekete-Szegö and Zalcman functionals for various subclasses of univalent and multivalent functions.

Throughout this paper it is assumed that functions $\phi$ and $h$ are analytic in U. Also let

$$
\phi(z)=A_{0}+A_{1} z+A_{2} z^{2}+\cdots \quad(|\phi(z)| \leq 1, z \in \mathbb{U})
$$

and

$$
\mathrm{h}(z)=1+\mathrm{B}_{1} z+\mathrm{B}_{2} z^{2}+\cdots \quad\left(\mathrm{B}_{1} \in \mathbb{R}^{+}\right) .
$$

Motivated by earlier works in ([6], [7], [15], [17], [19]) on quasi-subordination, we introduce here the following subclass of analytic functions:

Definition 1 For $0 \leq \lambda \leq 1$ and $\gamma \in \mathbb{C} \backslash\{0\}$, a function $\mathrm{f} \in \mathcal{A}$ given by (1) is said to be in the class $\mathcal{K}_{\mathrm{q}}(\lambda, \gamma, \mathrm{h})$ if the following condition are satisfied:

$$
\frac{1}{\gamma}\left(\frac{z f^{\prime}(z)+z^{2} f^{\prime \prime}(z)}{(1-\lambda) z+\lambda z f^{\prime}(z)}-1\right) \prec_{q}(h(z)-1),
$$

where $\mathrm{h}$ is given by (3) and $z \in \mathbb{U}$.

It follows that a function $\mathrm{f}$ is in the class $\mathcal{K}_{\mathrm{q}}(\lambda, \gamma, \mathrm{h})$ if and only if there exists an analytic function $\phi$ with $|\phi(z)| \leq 1$, in $\mathbb{U}$ such that

$$
\frac{\frac{1}{\gamma}\left(\frac{z f^{\prime}(z)+z^{2} f^{\prime \prime}(z)}{(1-\lambda) z+\lambda z f^{\prime}(z)}-1\right)}{\phi(z)} \prec(h(z)-1)
$$

where $\boldsymbol{h}$ is given by (3) and $z \in \mathbb{U}$.

If we set $\phi(z) \equiv 1(z \in \mathbb{U})$, then the class $\mathcal{K}_{\mathrm{q}}(\lambda, \gamma, h)$ is denoted by $\mathcal{K}(\lambda, \gamma, h)$ satisfying the condition that

$$
1+\frac{1}{\gamma}\left(\frac{z f^{\prime}(z)+z^{2} f^{\prime \prime}(z)}{(1-\lambda) z+\lambda z f^{\prime}(z)}-1\right) \prec h(z) \quad(z \in \mathbb{U}) .
$$

In the present paper, we find sharp bounds on the Fekete-Szegö functional for functions belonging in the class $\mathcal{K}_{\mathrm{q}}(\lambda, \gamma, \mathrm{h})$. Several known and new consequences of these results are also pointed out. In order to derive our main results, we have to recall here the following well-known lemma: 
Let $\Omega$ be class of analytic functions of the form

$$
w(z)=w_{1} z+w_{2} z^{2}+\cdots
$$

in the unit disk $\mathbb{U}$ satisfying the condition $|w(z)|<1$.

Lemma 1 ([8], p.10) If $w \in \Omega$, then for any complex number $v$ :

$$
\left|w_{1}\right| \leq 1,\left|w_{2}-v w_{1}^{2}\right| \leq 1+(|v|-1)\left|w_{1}^{2}\right| \leq \max \{1,|v|\} .
$$

The result is sharp for the functions $w(z)=z$ or $w(z)=z^{2}$.

\section{Main results}

Theorem 1 Let $0 \leq \lambda \leq 1$ and $\gamma \in \mathbb{C} \backslash\{0\}$. If $\mathrm{f} \in \mathcal{A}$ of the form (1) belonging to the class $\mathcal{K}_{\mathrm{q}}(\lambda, \gamma, \mathrm{h})$, then

$$
\left|\mathrm{a}_{2}\right| \leq \frac{|\gamma| \mathrm{B}_{1}}{2(2-\lambda)}
$$

and for any $\vee \in \mathbb{C}$

$$
\left|\mathrm{a}_{3}-v \mathrm{a}_{2}^{2}\right| \leq \frac{|\gamma| \mathrm{B}_{1}}{3(3-\lambda)} \max \left\{1,\left|\frac{\mathrm{B}_{2}}{\mathrm{~B}_{1}}-\mathrm{QB}_{1}\right|\right\},
$$

where

$$
\mathrm{Q}=\gamma\left(\frac{3 v(3-\lambda)}{4(2-\lambda)^{2}}-\frac{\lambda}{2-\lambda}\right)
$$

The results are sharp.

Proof. Let $f \in \mathcal{K}_{q}(\lambda, \gamma, h)$. In view of Definition 1, there exist then Schwarz functions $w$ and an analytic function $\phi$ such that

$$
\frac{1}{\gamma}\left(\frac{z f^{\prime}(z)+z^{2} f^{\prime \prime}(z)}{(1-\lambda) z+\lambda z f^{\prime}(z)}-1\right)=\phi(z)(h(w(z))-1) \quad(z \in \mathbb{U}) .
$$

Series expansions for $f$ and its successive derivatives from (1) gives us

$$
\frac{1}{\gamma}\left(\frac{z f^{\prime}(z)+z^{2} f^{\prime \prime}(z)}{(1-\lambda) z+\lambda z f^{\prime}(z)}-1\right)=\frac{1}{\gamma}\left[2(2-\lambda) a_{2} z+\left(3(3-\lambda) a_{3}-4 \lambda(2-\lambda) a_{2}^{2}\right) z^{2}+\ldots\right] .
$$


Similarly from (2), (3) and (5), we obtain

$$
h(w(z))-1=\mathrm{B}_{1} w_{1} z+\left(\mathrm{B}_{1} w_{2}+\mathrm{B}_{2} w_{1}^{2}\right) z^{2}+\cdots
$$

and

$$
\phi(z)(h(w(z))-1)=A_{0} B_{1} w_{1} z+\left[A_{1} B_{1} w_{1}+A_{0}\left(B_{1} w_{2}+B_{2} w_{1}^{2}\right)\right] z^{2}+\cdots .
$$

Equating (10) and (11) in view of (9) and comparing the coefficients of $z$ and $z^{2}$, we get

$$
a_{2}=\frac{\gamma A_{0} B_{1} w_{1}}{2(2-\lambda)}
$$

and

$$
a_{3}=\frac{\gamma B_{1}}{3(3-\lambda)}\left[A_{1} w_{1}+A_{0}\left\{w_{2}+\left(\frac{\gamma \lambda A_{0} B_{1}}{2-\lambda}+\frac{B_{2}}{B_{1}}\right) w_{1}^{2}\right\}\right] .
$$

Thus, for any $v \in \mathbb{C}$, we have

$$
\begin{gathered}
a_{3}-v a_{2}^{2}=\frac{\gamma B_{1}}{3(3-\lambda)}\left[A_{1} w_{1}+\left(w_{2}+\frac{B_{2}}{B_{1}} w_{1}^{2}\right) A_{0}-\left(\frac{3(3-\lambda) \gamma}{4(2-\lambda)^{2}} v-\frac{\gamma \lambda}{2-\lambda}\right) B_{1} A_{0}^{2} w_{1}^{2}\right] \\
=\frac{\gamma B_{1}}{3(3-\lambda)}\left[A_{1} w_{1}+\left(w_{2}+\frac{B_{2}}{B_{1}} w_{1}^{2}\right) A_{0}-Q_{1} A_{0}^{2} w_{1}^{2}\right]
\end{gathered}
$$

where $\mathrm{Q}$ is given by $(8)$.

Since $\phi(z)=A_{0}+A_{1} z+A_{2} z^{2}+\cdots$ is analytic and bounded by one in $\mathbb{U}$, therefore we have (see [16], p 172 )

$$
\left|A_{0}\right| \leq 1 \text { and } A_{1}=\left(1-A_{0}^{2}\right) y \quad(y \leq 1) .
$$

From (14) and (15), we obtain

$$
a_{3}-v a_{2}^{2}=\frac{\gamma B_{1}}{3(3-\lambda)}\left[y w_{1}+\left(w_{2}+\frac{B_{2}}{B_{1}} w_{1}^{2}\right) A_{0}-\left(B_{1} Q w_{1}^{2}+y w_{1}\right) A_{0}^{2}\right] .
$$

If $A_{0}=0$ in (16), we at once get

$$
\left|a_{3}-v a_{2}^{2}\right| \leq \frac{|\gamma| B_{1}}{3(3-\lambda)}
$$

But if $A_{0} \neq 0$, let us then suppose that

$$
G\left(A_{0}\right)=y w_{1}+\left(w_{2}+\frac{B_{2}}{B_{1}} w_{1}^{2}\right) A_{0}-\left(B_{1} Q w_{1}^{2}+y w_{1}\right) A_{0}^{2}
$$


which is a quadratic polynomial in $A_{0}$ and hence analytic in $\left|A_{0}\right| \leq 1$ and maximum value of $\left|G\left(A_{0}\right)\right|$ is attained at $A_{0}=e^{\imath \theta}(0 \leq \theta<2 \pi)$, we find that

$$
\begin{aligned}
\max \left|\mathrm{G}\left(\mathrm{A}_{0}\right)\right| & =\max _{0 \leq \theta<2 \pi}\left|\mathrm{G}\left(\mathrm{e}^{\mathrm{\ell} \theta}\right)\right|=|\mathrm{G}(1)| \\
& =\left|w_{2}-\left(\mathrm{QB}_{1}-\frac{\mathrm{B}_{2}}{\mathrm{~B}_{1}}\right) w_{1}^{2}\right| .
\end{aligned}
$$

Therefore, it follows from (16) that

$$
\left|\mathrm{a}_{3}-v \mathrm{a}_{2}^{2}\right| \leq \frac{|\gamma| \mathrm{B}_{1}}{3(3-\lambda)}\left|w_{2}-\left(\mathrm{QB}_{1}-\frac{\mathrm{B}_{2}}{\mathrm{~B}_{1}}\right) w_{1}^{2}\right|,
$$

which on using Lemma 1, shows that

$$
\left|\mathrm{a}_{3}-v \mathrm{a}_{2}^{2}\right| \leq \frac{|\gamma| \mathrm{B}_{1}}{3(3-\lambda)} \max \left\{1,\left|\frac{\mathrm{B}_{2}}{\mathrm{~B}_{1}}-\mathrm{QB}_{1}\right|\right\},
$$

and this last above inequality together with (17) establish the results. The result are sharps for the function $f$ given by

$$
\begin{aligned}
& 1+\frac{1}{\gamma}\left(\frac{z f^{\prime}(z)+z^{2} f^{\prime \prime}(z)}{(1-\lambda) z+\lambda z f^{\prime}(z)}-1\right)=h(z), \\
& 1+\frac{1}{\gamma}\left(\frac{z f^{\prime}(z)+z^{2} f^{\prime \prime}(z)}{(1-\lambda) z+\lambda z f^{\prime}(z)}-1\right)=h\left(z^{2}\right)
\end{aligned}
$$

and

$$
1+\frac{1}{\gamma}\left(\frac{z f^{\prime}(z)+z^{2} f^{\prime \prime}(z)}{(1-\lambda) z+\lambda z f^{\prime}(z)}-1\right)=z(h(z)-1) .
$$

This completes the proof of Theorem 1 .

For $\lambda=0$ the Theorem 1 reduces to following corollary:

Corollary 1 If $\mathrm{f} \in \mathcal{A}$ of the form (1) satisfies

$$
\frac{1}{\gamma}\left(f^{\prime}(z)+z f^{\prime \prime}(z)-1\right) \prec_{\mathrm{q}}(\mathrm{h}(z)-1) \quad(z \in \mathbb{U}, \gamma \in \mathbb{C} \backslash\{0\}),
$$

then

$$
\left|\mathrm{a}_{2}\right| \leq \frac{|\gamma| \mathrm{B}_{1}}{4}
$$

and for some $\boldsymbol{v} \in \mathbb{C}$

$$
\left|\mathrm{a}_{3}-v \mathrm{a}_{2}^{2}\right| \leq \frac{|\gamma| \mathrm{B}_{1}}{9} \max \left\{1,\left|\frac{\mathrm{B}_{2}}{\mathrm{~B}_{1}}-\frac{9 v|\gamma| \mathrm{B}_{1}}{16}\right|\right\} .
$$

The results are sharp. 
Remark 1 In Corollary 1 , if we set $\phi \equiv 1$, then above result match with the result given in [3].

Remark 2 For $\phi \equiv 1, \gamma=\lambda=1$, Theorem 1 reduces to an improved result of given in [15].

The next theorem gives the result based on majorization.

Theorem 2 Let $0 \leq \lambda \leq 1$ and $\gamma \in \mathbb{C} \backslash\{0\}$. If $\mathrm{f} \in \mathcal{A}$ of the form (1) satisfies

$$
\frac{1}{\gamma}\left(\frac{z f^{\prime}(z)+z^{2} f^{\prime \prime}(z)}{(1-\lambda) z+\lambda z f^{\prime}(z)}-1\right) \ll(h(z)-1) \quad(z \in \mathbb{U}),
$$

then

$$
\left|a_{2}\right| \leq \frac{|\gamma| B_{1}}{2(2-\lambda)}
$$

and for any $\vee \in \mathbb{C}$

$$
\left|\mathrm{a}_{3}-v \mathrm{a}_{2}^{2}\right| \leq \frac{|\gamma| \mathrm{B}_{1}}{3(3-\lambda)} \max \left\{1,\left|\frac{\mathrm{B}_{2}}{\mathrm{~B}_{1}}-\mathrm{QB}_{1}\right|\right\}
$$

where $Q$ is given by (8). The results are sharp.

Proof. Assume that (19) holds. From the definition of majorization, there exist an analytic function $\phi$ such that

$$
\frac{1}{\gamma}\left(\frac{z f^{\prime}(z)+z^{2} f^{\prime \prime}(z)}{(1-\lambda) z+\lambda z f^{\prime}(z)}-1\right)=\phi(z)(h(z)-1) \quad(z \in \mathbb{U}) .
$$

Following similar steps as in the proof of Theorem 1 , and by setting $w(z) \equiv z$, so that $w_{1}=1, w_{n}=0, n \geq 2$, we obtain

$$
a_{2}=\frac{\gamma A_{0} B_{1}}{2(2-\lambda)}
$$

and also we obtain that

$$
a_{3}-v a_{2}^{2}=\frac{\gamma B_{1}}{3(3-\lambda)}\left[A_{1}+\frac{B_{2}}{B_{1}} A_{0}-Q_{1} A_{0}^{2}\right] .
$$

On putting the value of $A_{1}$ from (15), we obtain

$$
a_{3}-v a_{2}^{2}=\frac{\gamma B_{1}}{3(3-\lambda)}\left[y+\frac{B_{2}}{B_{1}} A_{0}-\left(Q_{1}+y\right) A_{0}^{2}\right] .
$$


If $A_{0}=0$ in $(20)$, we at once get

$$
\left|a_{3}-v a_{2}^{2}\right| \leq \frac{|\gamma| B_{1}}{3(3-\lambda)},
$$

But if $A_{0} \neq 0$, let us then suppose that

$$
T\left(A_{0}\right)=y+\frac{B_{2}}{B_{1}} A_{0}-\left(Q_{1}+y\right) A_{0}^{2},
$$

which is a quadratic polynomial in $A_{0}$, hence analytic in $\left|A_{0}\right| \leq 1$ and maximum value of $\left|T\left(A_{0}\right)\right|$ is attained at $A_{0}=e^{i \theta}(0 \leq \theta<2 \pi)$, we find that

$$
\max \left|T\left(A_{0}\right)\right|=\max _{0 \leq \theta<2 \pi}\left|T\left(e^{\iota \theta}\right)\right|=|T(1)| .
$$

Hence, from (20), we obtain

$$
\left|\mathrm{a}_{3}-v \mathrm{a}_{2}^{2}\right| \leq \frac{|\gamma| \mathrm{B}_{1}}{3(3-\lambda)}\left|\mathrm{QB}_{1}-\frac{\mathrm{B}_{2}}{\mathrm{~B}_{1}}\right| .
$$

Thus, the assertion of Theorem 2 follows from this last above inequality together with (21). The results are sharp for the function given by

$$
1+\frac{1}{\gamma}\left(\frac{z f^{\prime}(z)+z^{2} f^{\prime \prime}(z)}{(1-\lambda) z+\lambda z f^{\prime}(z)}-1\right)=h(z),
$$

which completes the proof of Theorem 2 .

Theorem 3 Let $0 \leq \lambda \leq 1$ and $\gamma \in \mathbb{C} \backslash\{0\}$. If $\mathrm{f} \in \mathcal{A}$ of the form (1) belonging to the class $\mathcal{K}(\lambda, \gamma, \mathrm{h})$, then

$$
\left|\mathrm{a}_{2}\right| \leq \frac{|\gamma| \mathrm{B}_{1}}{2(2-\lambda)}
$$

and for any $v \in \mathbb{C}$

$$
\left|\mathrm{a}_{3}-v \mathrm{a}_{2}^{2}\right| \leq \frac{|\gamma| \mathrm{B}_{1}}{3(3-\lambda)} \max \left\{1,\left|\frac{\mathrm{B}_{2}}{\mathrm{~B}_{1}}-\mathrm{QB}_{1}\right|\right\},
$$

where $Q$ is given by (8), the results are sharp. 
Proof. The proof is similar to Theorem 1 , Let $f \in \mathcal{K}(\lambda, \gamma, \mathrm{h})$.

If $\phi(z)=1$, then $A_{0}=1, A_{n}=0(n \in \mathbb{N})$. Therefore, in view of (12) and (14) and by application of Lemma 1, we obtain the desired assertion. The results are sharp for the function $f$ given by

$$
1+\frac{1}{\gamma}\left(\frac{z f^{\prime}(z)+z^{2} f^{\prime \prime}(z)}{(1-\lambda) z+\lambda z f^{\prime}(z)}-1\right)=h(z),
$$

or

$$
1+\frac{1}{\gamma}\left(\frac{z f^{\prime}(z)+z^{2} f^{\prime \prime}(z)}{(1-\lambda) z+\lambda z f^{\prime}(z)}-1\right)=h\left(z^{2}\right) .
$$

Thus, the proof of Theorem 3 is completed.

Now, we determine the bounds on the functional $\left|a_{3}-v a_{2}^{2}\right|$ for real $v$.

Theorem 4 Let $0 \leq \lambda \leq 1$. If $\mathrm{f} \in \mathcal{A}$ of the form (1) belonging to the class $\mathcal{K}_{\mathrm{q}}(\lambda, \gamma, \mathrm{h})$, then for real $\boldsymbol{\nu}$ and $\gamma$, we have

$$
\left|\mathrm{a}_{3}-v \mathrm{a}_{2}^{2}\right| \leq \begin{cases}\frac{|\gamma| \mathrm{B}_{1}}{3(3-\lambda)}\left[\mathrm{B}_{1} \gamma\left(\frac{\lambda}{2-\lambda}-\frac{3(3-\lambda)}{4(2-\lambda)^{2}} v\right)+\frac{\mathrm{B}_{2}}{\mathrm{~B}_{1}}\right] & \left(v \leq \sigma_{1}\right), \\ \frac{|\gamma| \mathrm{B}_{1}}{3(3-\lambda)} & \left(\sigma_{1} \leq v \leq \sigma_{1}+2 \rho\right) \\ -\frac{|\gamma| \mathrm{B}_{1}}{3(3-\lambda)}\left[\mathrm{B}_{1} \gamma\left(\frac{\lambda}{2-\lambda}-\frac{3(3-\lambda)}{4(2-\lambda)^{2}} v\right)+\frac{\mathrm{B}_{2}}{\mathrm{~B}_{1}}\right] & \left(v \geq \sigma_{1}+2 \rho\right)\end{cases}
$$

where

$$
\sigma_{1}=\frac{4 \lambda(2-\lambda)}{3(3-\lambda)}-\frac{4(2-\lambda)^{2}}{3 \gamma(3-\lambda)}\left(\frac{1}{B_{1}}-\frac{B_{2}}{B_{1}^{2}}\right)
$$

and

$$
\rho=\frac{4(2-\lambda)^{2}}{3 \gamma(3-\lambda) B_{1}} .
$$

Each of the estimates in (22) are sharp.

Proof. For real values of $v$ and $\gamma$ the above bounds can be obtained from (7), respectively, under the following cases:

$$
\mathrm{B}_{1} \mathrm{Q}-\frac{\mathrm{B}_{2}}{\mathrm{~B}_{1}} \leq-1,-1 \leq \mathrm{B}_{1} \mathrm{Q}-\frac{\mathrm{B}_{2}}{\mathrm{~B}_{1}} \leq 1 \text { and } \mathrm{B}_{1} \mathrm{Q}-\frac{\mathrm{B}_{2}}{\mathrm{~B}_{1}} \geq 1
$$

where $\mathrm{Q}$ is given by (8). We also note the following:

(i) When $v<\sigma_{1}$ or $v>\sigma_{1}+2 \rho$, then the equality holds if and only if $\phi(z) \equiv 1$ and $w(z)=z$ or one of its rotations. 
(ii) When $\sigma_{1}<v<\sigma_{1}+2 \rho$, then the equality holds if and only if $\phi(z) \equiv 1$ and $w(z)=z^{2}$ or one of its rotations.

(iii) Equality holds for $v=\sigma_{1}$ if and only if $\phi(z) \equiv 1$ and $w(z)=\frac{z(z+\epsilon)}{1+\epsilon z}(0 \leq$ $\epsilon \leq 1$ ), or one of its rotations, while for $v=\sigma_{1}+2 \rho$, the equality holds if and only if $\phi(z) \equiv 1$ and $w(z)=-\frac{z(z+\epsilon)}{1+\epsilon z}(0 \leq \epsilon \leq 1)$, or one of its rotations.

The bounds of the functional $a_{3}-v a_{2}^{2}$ for real values of $v$ and $\gamma$ for the middle range of the parameter $v$ can be improved further as follows:

Theorem 5 Let $0 \leq \lambda \leq 1$. If $\mathrm{f} \in \mathcal{A}$ of the form (1) belonging to the class $\mathcal{K}_{\mathrm{q}}(\lambda, \gamma, \mathrm{h})$, then for real $\boldsymbol{\nu}$ and $\gamma$, we have

$$
\left|a_{3}-v a_{2}^{2}\right|+\left(v-\sigma_{1}\right)\left|a_{2}\right|^{2} \leq \frac{|\gamma| B_{1}}{3(3-\lambda)} \quad\left(\sigma_{1} \leq v \leq \sigma_{1}+\rho\right)
$$

and

$$
\left|a_{3}-v a_{2}^{2}\right|+\left(\sigma_{1}+2 \rho-v\right)\left|a_{2}\right|^{2} \leq \frac{|\gamma| B_{1}}{3(3-\lambda)} \quad\left(\sigma_{1}+\rho \leq v \leq \sigma_{1}+2 \rho\right),
$$

where $\sigma_{1}$ and $\rho$ are given by (23) and (24), respectively.

Proof. Let $f \in \mathcal{K}_{\mathrm{q}}(\lambda, \gamma, h)$. For real $v$ satisfying $\sigma_{1}+\rho \leq v \leq \sigma_{1}+2 \rho$ and using (12) and (18) we get

$\left|a_{3}-v a_{2}^{2}\right|+\left(v-\sigma_{1}\right)\left|a_{2}\right|^{2}$

$\leq \frac{|\gamma| \mathrm{B}_{1}}{3(3-\lambda)}\left[\left|w_{2}\right|-\frac{3|\gamma| \mathrm{B}_{1}(3-\lambda)}{4(2-\lambda)^{2}}\left(v-\sigma_{1}-\rho\right)\left|w_{1}\right|^{2}+\frac{3|\gamma| \mathrm{B}_{1}(3-\lambda)}{4(2-\lambda)^{2}}\left(\nu-\sigma_{1}\right)\left|w_{1}\right|^{2}\right]$.

Therefore, by virtue of Lemma 1 , we get

$$
\left|a_{3}-v a_{2}^{2}\right|+\left(v-\sigma_{1}\right)\left|a_{2}\right|^{2} \leq \frac{|\gamma| B_{1}}{3(3-\lambda)}\left[1-\left|w_{1}\right|^{2}+\left|w_{1}\right|^{2}\right],
$$

which yields the assertion (25).

If $\sigma_{1}+\rho \leq v \leq \sigma_{1}+2 \rho$, then again from (12), (18) and the application of Lemma 1 , we have

$$
\begin{aligned}
\left|\mathrm{a}_{3}-v \mathrm{a}_{2}^{2}\right|+\left(\sigma_{1}+2 \rho-v\right)\left|\mathrm{a}_{2}\right|^{2} \leq & \frac{|\gamma| \mathrm{B}_{1}}{3(3-\lambda)}\left[\left|w_{2}\right|+\frac{3|\gamma| \mathrm{B}_{1}(3-\lambda)}{4(2-\lambda)^{2}}\left(v-\sigma_{1}-\rho\right)\left|w_{1}\right|^{2}\right. \\
& \left.+\frac{3|\gamma| \mathrm{B}_{1}(3-\lambda)}{4(2-\lambda)^{2}}\left(\sigma_{1}+2 \rho-v\right)\left|w_{1}\right|^{2}\right] \\
\leq & \frac{|\gamma| \mathrm{B}_{1}}{3(3-\lambda)}\left[1-\left|w_{1}\right|^{2}+\left|w_{1}\right|^{2}\right]
\end{aligned}
$$


which estimates (26).

\section{Conflicts of interest}

The authors declare that there are no conflict of interest regarding the publication of this paper.

\section{References}

[1] Y. Abu Muhanna, L. Li, S. Ponnusamy, Extremal problems on the class of convex functions of order -1/2, Arch. Math. (Basel), 103 (6) (2014), 461-471.

[2] R.M. Ali, V Ravichandran, N. Seenivasagan, Coefficient bounds for $p$ valent functions, Appl. Math. Comput., 187 (2007), 35-46.

[3] D. Bansal, Fekete-Szegő Problem for a New Class of Analytic Functions, Int. J. Math. Math. Sci., Article ID 143096 (2011), 5 pages.

[4] J. E. Brown, A. Tsao, On the Zalcman conjecture for starlike and typically real functions, Math. Z., 191 (1986), 467-474.

[5] M. Fekete, G. Szegő, Eine Bemerkung Über ungerade schlichte Funktionen, J. London Math. Soc., 8 (1933), 85-89.

[6] S. P. Goyal, O. Singh, Fekete-Szegő problems and coefficient estimates of quasi-subordination classes, J. Rajasthan Acad. Phys. Sci., 13 (2) (2014), $133-142$.

[7] S. Kant, Coefficients estimate for certain subclasses of bi-univalent functions associated with quasi-subordination, J. Fract. Calc. Appl., 9 (1) (2018), 195-203.

[8] F. R. Keogh, E. P. Merkes, A coefficient inequality for certain classes of analytic functions, Proc. Amer. Math. Soc., 20 (1969), 8-12.

[9] W. Koepf, On the Fekete-Szegö problem for close-to-convex functions, Proc. Amer. Math. Soc., 101 (1) (1987), 89-95.

[10] W. Koepf, On the Fekete-Szegő problem for close-to-convex functions II, Arch. Math. (Basel), 49 (5) (1987), 420-433. 
[11] S. L. Krushkal, Proof of the Zalcman conjecture for initial coefficients, Georgian Math. J., 17 (4) (2010), 663-681.

[12] L. Li, S. Ponnusamy, On the genralized Zalcman functional $\lambda a_{n}^{2}-a_{2 n-1}$ in the close-to-convex family, Proc. Amer. Math. Soc., 145, (2) (2017), 833-846.

[13] L. Li, S. Ponnusamy, J. Qiao, Generalized Zalcman conjecture for convex functions of order $\alpha$, Acta Math. Hungar., 150 (1) (2016), 234-246.

[14] W. Ma, Generalized Zalcman conjecture for starlike and typically real functions, J. Math. Anal. Appl., 234 (1999), 328-339.

[15] M. H. Mohd, M. Darus, Fekete-Szegő problems for quasi-subordination classes, Abstr. Appl. Anal., Article ID 192956 (2012), 14 pages.

[16] Z. Nehari, Conformal mapping, Dover, New York (1975) (reprinting of the 1952 edition).

[17] T. Panigrahi, R. K. Raina, Fekete-Szegő coefficient functional for quasisubordination class, Afro. Mat., 28 (5-6) (2017), 707-716.

[18] M. S. Robertson, Quasi-subordination and coefficient conjuctures, Bull. Amer. Math. Soc., 76 (1970), 1-9.

[19] P. Sharma, R. K. Raina, On a Sakaguchi type class of analytic functions associated with quasisubordination, Comment. Math. Univ. St. Pauli, 64 (1) (2015), 59-70.

[20] H. M. Srivastava, A. K. Mishra, M. K. Das, The Fekete-Szegő problem for a subclass of close-to-convex functions, Complex Var. Theory Appl., 44 (2001), 145-163. 